\title{
Pancreatic Function
}

Several papers published in Gastrcenterology, vol. 11, November, 1948, give interesting reports cf studies in pancreatic function. In the first, by Dornberger, Comfort, Wcllaeger and Power (p. 691), analyses of faeces for total fat is shown to give information of value in the early diagnosis of pancreatitis. This is more valuable than analysis for total faecal solids and faecal nitrogen, and in some cases provides data diagnostic of chronic pancreatitis. Intake-excretion studies were carried out in 20 cases of chronic pancreatitis, in ten of which marked evidence of proved pancreatic damage was present. The amount of loss of fat in the stools was roughly in proportion to the degree of pancreatic damage, and the percentage of ingested fat loss ranged up to approximately 44 per cent. Abnormal loss of protein did not always accompany abnormal steatorrhoea, which in the severe cases was more than five times the normal loss.

Further studies using the secretin test, are reported by Dornbergsr and Power (p. 701), and by Dreíling and Hollander (p. 714). This test is carried out by using a double lumen tube introduced into the stomach and duodenum. The tube's position is checked by X-ray and continuous aspiration is then kept up. The duodenal contents are collected and any overflow of gastric juice into the duodenum is prevented by constant aspiration. After an initial $\mathrm{p}$ re-stimulation period of aspiration purified secretin is given intraven usly in dose of one clinical unit per kilogram. Studies of the pancreatic secretion are then carried out, estimating both the volume of duodenal contents, Enzyme activity and in particular the bicarbonate concentration. The studies reported consist of an analysis of this test carried out on 28 cases of chronic pancreatitis, and in the second paper on some 145 cases of normal individuals and patients suffering from pancreatic carcinoma, post-cholecystectomy syndrome and jaundice. In the first group analysis of the duodenal contents before and after stimulation with secretin showed that in patients with pancreatitis the volume of secretion after stimulation was in general less than the minimum obtained per normal person, and the

Editorial

361

average concentration of bicarbonate was also lower. At the same time the sustained secretion of bicarbonate was less roughly in proportion to the degree of damage to the pancreas. In contrast to this the enxymes in general are less affected, though in cases where the pancreas is badly diseased they tend to be fixed at low concentrations; this, however, was rarely sufficient to disclose evidence of disease. Analysis of the mean values for amylase and trypsin were less significant than the values for lipase, and statistical analysis of the data from normal people showed that the variability of values for total enzymes is so large that a value in an individual case can be considered presumptively abnormal ronly if it is zero or very low; the ranges -of values however for volume and for concentration of bicarbonate tand total bicarbonate as disclosed by the standard deviation iare relatively well defined at a level considerably above zero. These findings confirm the knowledge that pure ,secretin is an active stimulant of secretion of water and bicarbonate, and merely washes out the enzymes. It appears that active secretion of the enzymes is in response to stimulation of the vagus and to pancreo/.ymin. Determinations of 
total enzymes were of no assistance in diagnosis, and in no case did determination of enzyme concentration add to information obtained from determination of duodenal volume and bicarbonate concentration,

The Authors conclude from these studies that determination of enzymes might well be deleted from the test without impairing its value. They believed that this Test disclosed insufficiency of external secretion more often and appears to be of greater value in the diagnosis of chronic pancreatitis than does faecal analysis.

In a few other preliminary studies by the seerelin test Drei-ling and Hollander found some points of interest, in particular in the "post-cholecystoctomy" syndrome which often gives rise to much clinical difficulty in diagnosis. They suggest that the test may disclose those cases in which incomplete common duct obstruction persists, and may also, of course, assist in excluding pancreatic disease. l"ests carried out in cases of jaundice combining the seeretiin volume response with curves of bile pigment in the duodenal secretion were somewhat equivocal, but further studies on these lines may, it is hoped, lead to help in the differential diagnosis of carcinoma of the pancreas, hepatitis, cirrhosis and gall-stone obstruction. 